\title{
EVALUATION OF POST-BUCKLING BEHAVIOR OF UNSYMMETRICAL COMPRESSIVE DELAMINATION IN CYLINDRICAL COMPOSITE PANELS OF AIRCRAFT STRUCTURES
}

\author{
Oleg Mitrofanov ${ }^{1}$, Igors Lebedevs ${ }^{2}$, Vladislavs Turko ${ }^{2}$ \\ ${ }^{1}$ Moscow Aviation Institute (National Research University), Russia; \\ ${ }^{2}$ Riga Technical University, Latvia \\ mitrofanovov@mai.ru,igors.lebedevs@aviatest.lv, vladislav.turko@gmail.com \\ mitrofanovov@mai.ru,igors.lebedevs@aviatest.lv, margarita.urbaha@rtu.lv
}

\begin{abstract}
The research of surface defects, such as delaminations in elements of thin-walled aircraft structures made of composite materials, is a topical problem. The paper considers an analytical solution to the problem of the overcritical behaviour of thin delaminations close to one of the surfaces in composite panels with surface curvature. The objects of research in this case can be nose parts of composite mechanization units, as well as semicircular specimens that allow laboratory research of these types of defects. Studies on laboratory cylindrical specimens with defects make it possible to determine the allowable dimensions of the defects, as well as the maximum allowable level of loading at which it is impossible for the defects to propagate. In accordance with modern aviation regulations, it is not allowed for defects to propagate (stagger) even under the calculated level of loading. The presented initial relations of the geometrically nonlinear problem consider the possible asymmetric structure of the delamination along the thickness as well as the small curvature of the studied panels. The type of deflection in this case of the delamination corresponds to a rigid support. When solving the problem by the Bubnov-Galerkin method, the stress function and expressions for the corresponding membrane stresses under possible overcritical behaviour of the stratification are obtained. A nonlinear expression linking the deflection amplitude and the compressive force is also written. When considering a linear problem, an analytical expression for compressive critical stresses is obtained. In the general case, the obtained expressions consider the arrangement of composite layers along the thickness, as well as the asymmetric structure of the delamination. Examples of determination of critical forces for panels with asymmetric structure are given.
\end{abstract}

Keywords: post-buckling state, composite material, asymmetric structure, cylindrical panel, delamination, compression.

\section{Introduction}

When designing elements of modern aircraft structures made of composite materials (CM), it is necessary to consider the possible presence of defects at all stages of the life cycle of the aircraft $[1 ; 2]$. When investigating defects in the elements of low- and medium-capacity aircraft structures, it is necessary to consider the possibility of the appearance of delaminations with different structures, including asymmetrical stacking. In this paper we consider the geometrically nonlinear behaviour of thin surface defects of the delamination type, considering the possible curvature of the supporting panels of the structure and the asymmetry of the structure.

In papers [3-4] the results of experimental studies of circumferential and semicircular thin-walled specimens made of CM are given. Principle scheme of tests [3-4] and calculated loading scheme for analytical estimation of post-buckling delamination behaviour in cylindrical panels are shown in Fig.1. In this paper we consider a geometrically nonlinear problem for a compressed thin layering considering the asymmetry of the structure. We will use the hypotheses of the theory of composite structures by V.V. Vasiliev [5], considering nonlinear geometric relations. Let us assume that the form of the splitting is close to square and that during compression one half-wave in the longitudinal and transverse directions is possible. The most correct boundary conditions correspond to rigid embedding.

Note that the results of the considered problem of studying the post-buckling behaviour of cylindrical composite panels can also be used for studies of the stress-strain state and verification calculations of thin composite shells of nose parts of light aircraft mechanization assemblies. In addition, analytical solutions to the stability and load-carrying capacity problems can be used to design cylindrical panels according to the post-buckling state. The methodology for the design of load-bearing composite panels is described in [6]. The computational and experimental results of studies considering the peculiarities of fracture mechanics and post-buckling behaviour of composite panels are also given in [7-10]. 


\section{Basic relationships and problem statement}

The initial relations for the considered problem are given in the monograph [5]. Let us write down the condition of joint deformations with consideration of geometrical nonlinearity and possible asymmetry of the structure in the form $[5 ; 11]$

$$
L_{1}(F)-L_{2}(W)+L_{5}(W)=0
$$

where

$$
\begin{gathered}
L_{1}(F)=c_{y} \frac{\partial^{4} F}{\partial x^{4}}+\left(c-2 c_{x y}\right) \frac{\partial^{4} F}{\partial x^{2} \partial y^{2}}+c_{x} \frac{\partial^{4} F}{\partial y^{4}}, \\
L_{2}(W)=\left(\frac{\partial^{2} W}{\partial x \partial y}\right)^{2}-\left(\frac{\partial^{2} W}{\partial x^{2}}\right)\left(\frac{\partial^{2} W}{\partial y^{2}}\right)-\frac{1}{R} \frac{\partial^{2} W}{\partial x^{2}},
\end{gathered}
$$
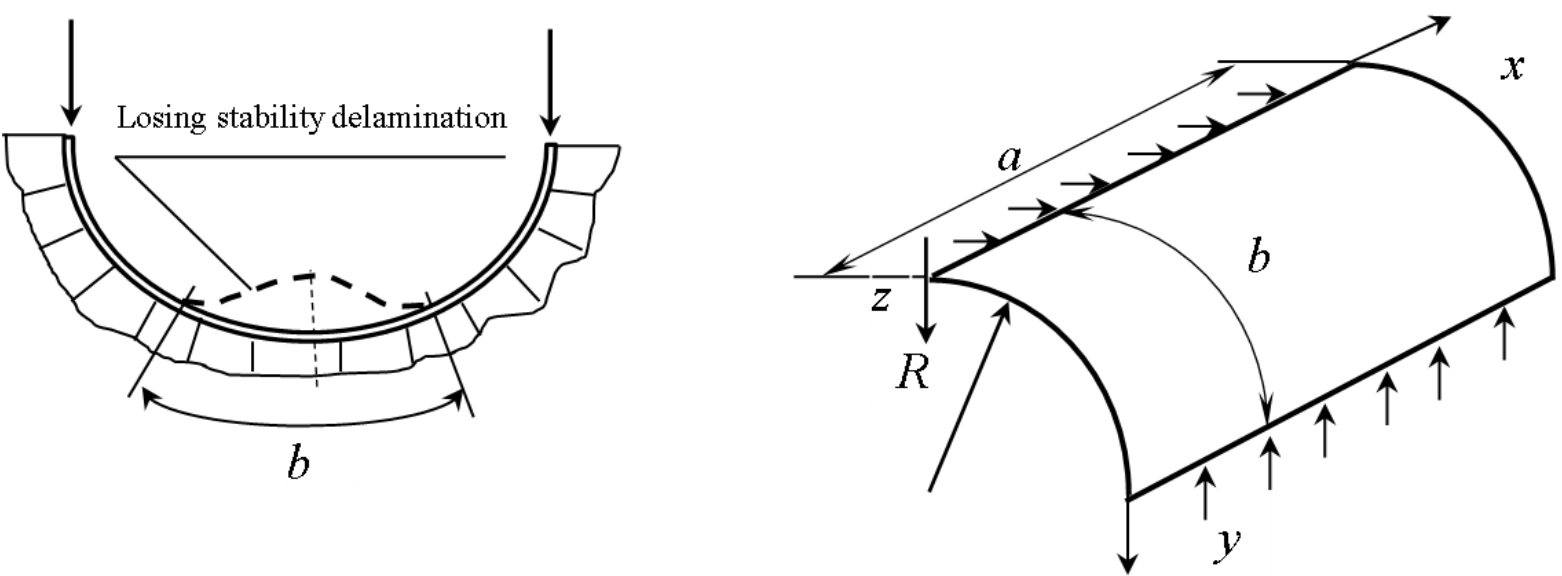

Fig.1. Load diagram of a half-ring specimen: a - with delamination [3; 4];

$\mathrm{b}$ - cylindrical panel (inverted view)

When considering the problems of stability and load-bearing capacity of cylindrical panels with an all-round rigid anchoring, let us represent the deflection (assuming that the positive direction of the deflection axis corresponds to the opposite direction of the $\mathrm{Z}$ axis) in the following form

$$
W=f \cdot \sin ^{2} \frac{\pi x}{a} \sin ^{2} \frac{\pi y}{b},
$$

where $\alpha=\pi / a$;

$\beta=\pi / b-$ waveform parameters.

Geometrically nonlinear Karman-type equations are written in the form $[5 ; 11]$

$$
L_{4}(W)-L_{3}(F, W)-L_{6}(F)=0,
$$

where

$$
\begin{gathered}
L_{4}(W)=D_{x} \frac{\partial^{4} W}{\partial x^{4}}+\left(4 D+D_{x y}+D_{y x}\right) \frac{\partial^{4} W}{\partial x^{2} \partial y^{2}}+D_{y} \frac{\partial^{4} W}{\partial y^{4}}, \\
\mathrm{~L}_{3}(\mathrm{~F}, \mathrm{~W})=\frac{\partial^{2} \mathrm{~F}}{\partial \mathrm{y}^{2}} \frac{\partial^{2} \mathrm{~W}}{\partial \mathrm{x}^{2}}+\frac{\partial^{2} \mathrm{~F}}{\partial \mathrm{x}^{2}} \frac{\partial^{2} \mathrm{~W}}{\partial \mathrm{y}^{2}}-2 \frac{\partial^{2} \mathrm{~F}}{\partial \mathrm{x} \partial \mathrm{y}} \frac{\partial^{2} \mathrm{~W}}{\partial \mathrm{x} \partial \mathrm{y}}+\frac{1}{R} \frac{\partial^{2} F}{\partial x^{2}}, \\
L_{6}(F)=C_{x y} \frac{\partial^{4} F}{\partial x^{4}}+2\left(C_{x}+C_{y}-2 C\right) \frac{\partial^{4} F}{\partial x^{2} \partial y^{2}}+C_{y x} \frac{\partial^{4} F}{\partial y^{4}} .
\end{gathered}
$$

The generalized stiffnesses of the laminated panel according to [5] are calculated by the relations

$$
B_{m n}=I_{m n}^{(0)}, C_{m n}=I_{m n}^{(1)}-e I_{m n}^{(0)}, D_{m n}=I_{m n}^{(2)}-2 e I_{m n}^{(1)}+e^{2} I_{m n}^{(0)},
$$




$$
I_{m n}^{(r)}=\frac{1}{r+1} \sum_{i=1}^{K} A_{m n}^{(i)}\left(t_{i}^{r+1}-t_{i-1}^{r+1}\right),(r=0,1,2), E_{11,22}=\frac{E_{1,2}}{1-\mu_{12} \mu_{21}},
$$

where

$$
\begin{gathered}
A_{11}^{(i)}=E_{11} \cos ^{4} \varphi_{i}+E_{22}^{(i)} \sin ^{4} \varphi_{i}+2\left[E_{11} \mu_{12}^{(i)}+2 G_{12}^{(i)}\right] \sin ^{2} \varphi_{i} \cos ^{2} \varphi_{i}, \\
A_{12}^{(i)}=A_{21}^{(i)}=E_{11} \mu_{12}^{(i)}+\left[E_{11}^{(i)}+E_{22}^{(i)}-2\left(E_{11} \mu_{12}^{(i)}+2 G_{12}^{(i)}\right)\right] \sin ^{2} \phi_{i} \cos ^{2} \phi_{i}, \\
A_{22}^{(i)}=E_{11} \sin ^{4} \varphi_{i}+E_{22} \cos ^{4} \varphi_{i}+2\left[E_{11} \mu_{12}^{(i)}+2 G_{12}^{(i)}\right] \sin ^{2} \varphi_{i} \cos ^{2} \varphi_{i}, \\
A_{33}^{(i)}=\left[E_{11}^{(i)}+E_{22}^{(i)}-2 E_{11}^{(i)} \mu_{12}^{(i)}\right] \sin ^{2} \varphi_{i} \cos ^{2} \varphi_{i}+G_{12}^{(i)} \cos ^{2} 2 \varphi_{i} .
\end{gathered}
$$

In addition, this paper retains the designations of the monograph [5]

$$
\begin{gathered}
c_{x}=\frac{B_{22}}{B}, c_{y}=\frac{B_{11}}{B}, c_{x y}=c_{y x}=\frac{B_{12}}{B}, c=\frac{1}{B_{33}}, B=B_{11} B_{22}-B_{12}^{2}, \\
B_{x}=\frac{1}{B}\left(B_{22} C_{11}-B_{12} C_{12}\right), B_{y}=\frac{1}{B}\left(B_{11} C_{22}-B_{12} C_{12}\right), \\
B_{x y}=\frac{1}{\mathrm{~B}}\left(B_{22} C_{12}-B_{12} C_{22}\right), B_{y x}=\frac{1}{B}\left(B_{11} C_{12}-B_{12} C_{11}\right), C=\frac{C_{33}}{B_{33}}, \\
C_{x}=c_{x}\left(C_{11}-\frac{c_{y x}}{c_{x}} C_{12}\right), C_{y}=c_{y}\left(C_{22}-\frac{c_{x y}}{c_{y}} C_{12}\right), \\
C_{x y}=c_{y}\left(C_{12}-\frac{c_{x y}}{c_{y}} C_{11}\right), C_{y x}=c_{x}\left(C_{12}-\frac{c_{y x}}{c_{x}} C_{22}\right), \\
D_{x}=D_{11}-C_{11} B_{x}-C_{12} B_{y x}, D_{y}=D_{22}-C_{22} B_{y}-C_{12} B_{x y}, \\
D_{x y}=D_{12}-C_{11} B_{x y}-C_{12} B_{y}, D_{y x}=D_{12}-C_{22} B_{y x}-C_{12} B_{x}, \\
D=\frac{D_{33} B_{33}-C_{33}^{2}}{B_{33}} .
\end{gathered}
$$

Membrane stresses of the panel that has lost stability are calculated using the definition of the stress function

$$
\sigma_{x}=\frac{1}{\delta} \frac{\partial^{2} F}{\partial y^{2}}, \sigma_{y}=\frac{1}{\delta} \frac{\partial^{2} F}{\partial x^{2}}, \tau_{x y}=-\frac{1}{\delta} \frac{\partial^{2} F}{\partial x \partial y}
$$

\section{Applied calculation methods}

To obtain an analytical solution of the geometrically nonlinear problem, we will use the BubnovGalerkin method.

After substituting the deflection (3) into the equality for $\mathrm{L} 2$ we can obtain the equation, the solution of which is a stress function

$$
\begin{gathered}
F=f^{2}\left\{A_{01} \cos \frac{2 \pi x}{a}+A_{02} \cos \frac{2 \pi y}{b}+A_{03} \cos \frac{2 \pi x}{a} \cos \frac{2 \pi y}{b}+\right. \\
\left.+A_{04} \cos \frac{2 \pi x}{a} \cos \frac{4 \pi y}{b}+A_{05} \cos \frac{4 \pi x}{a} \cos \frac{2 \pi y}{b}+A_{06} \cos \frac{4 \pi x}{a}+A_{07} \cos \frac{4 \pi y}{b}\right\}+ \\
+f\left\{t_{11} \cos \frac{2 \pi x}{a}+t_{12} \cos \frac{2 \pi y}{b}+t_{13} \cos \frac{2 \pi x}{a} \cos \frac{2 \pi y}{b}\right\}+\frac{N_{x} y^{2}}{2}+\frac{N_{y} x^{2}}{2},
\end{gathered}
$$




$$
\begin{gathered}
\text { where } \begin{array}{c}
A_{01}=\frac{a^{2}}{32 b^{2} c_{y}}, A_{02}=\frac{b^{2}}{32 a^{2} c_{x}}, A_{03}=-\frac{1}{16\left[c_{y}\left(\frac{b}{a}\right)^{2}+\left(c-2 c_{x y}\right)+c_{x}\left(\frac{a}{b}\right)^{2}\right]}, \\
A_{04}=\frac{1}{32\left[16 c_{y}\left(\frac{b}{a}\right)^{2}+4\left(c-2 c_{x y}\right)+c_{x}\left(\frac{a}{b}\right)^{2}\right]}, A_{05}=\frac{1}{32\left[c_{y}\left(\frac{b}{a}\right)^{2}+4\left(c-2 c_{x y}\right)+16 c_{x}\left(\frac{a}{b}\right)^{2}\right]}, \\
A_{06}=-\frac{1}{512 c_{y}}\left(\frac{a}{b}\right)^{2}, A_{07}=-\frac{1}{512 c_{x}}\left(\frac{b}{a}\right)^{2}, t_{11}=-\frac{B_{y x}}{4 c_{y}}-\frac{1}{R}\left(\frac{a}{\pi}\right)^{2} \frac{1}{c_{y}}, t_{12}=-\frac{B_{x y}}{4 c_{x}} \\
t_{13}=\frac{B_{y x} b^{4}+B_{x y} a^{4}+\left(B_{x}+B_{y}+2 C\right) a^{2} b^{2}}{4\left(c_{y} b^{4}+\left(c-2 c_{x y}\right) a^{2} b^{2}+c_{x} a^{4}\right)}+\frac{1}{R} \frac{1}{16\left(c_{y}\left(\frac{\pi}{a}\right)^{2}+\left(c-2 c_{x y}\right)\left(\frac{\pi}{b}\right)^{2}+c_{x}\left(\frac{a \pi}{b^{2}}\right)^{2}\right)} .
\end{array} .
\end{gathered}
$$

From the stress function (7) we obtain the normal stresses in the median surface, which has lost stability of the panel, under the action of the transverse force by the formulas

$$
\begin{gathered}
\sigma_{x}=\frac{f^{2}}{\delta}\left\{-\left(\frac{2 \pi y}{b}\right)^{2} A_{02} \cos \frac{2 \pi y}{b}-\left(\frac{2 \pi y}{b}\right)^{2} A_{03} \cos \frac{2 \pi x}{a} \cos \frac{2 \pi y}{b}-\right. \\
\left.-\left(\frac{4 \pi y}{b}\right)^{2} A_{04} \cos \frac{2 \pi x}{a} \cos \frac{4 \pi y}{b}-\left(\frac{2 \pi y}{b}\right)^{2} A_{05} \cos \frac{4 \pi x}{a} \cos \frac{2 \pi y}{b}-\left(\frac{4 \pi y}{b}\right)^{2} A_{07} \cos \frac{4 \pi y}{b}\right\}+ \\
+\frac{f}{\delta}\left\{-\left(\frac{2 \pi y}{b}\right)^{2} A_{12} \cos \frac{2 \pi y}{b}-\left(\frac{2 \pi y}{b}\right)^{2} A_{13} \cos \frac{2 \pi x}{a} \cos \frac{2 \pi y}{b}\right\},(8) \\
\sigma_{y}=\frac{f^{2}}{\delta}\left\{-\left(\frac{2 \pi}{a}\right)^{2} A_{01} \cos \frac{2 \pi x}{a}-\left(\frac{2 \pi}{a}\right)^{2} A_{03} \cos \frac{2 \pi x}{a} \cos \frac{2 \pi y}{b}-\right. \\
\left.-\left(\frac{2 \pi}{a}\right)^{2} A_{04} \cos \frac{2 \pi x}{a} \cos \frac{4 \pi y}{b}-\left(\frac{4 \pi}{a}\right)^{2} A_{05} \cos \frac{4 \pi x}{a} \cos \frac{2 \pi y}{b}-\left(\frac{4 \pi}{a}\right)^{2} A_{06} \cos \frac{4 \pi x}{a}\right\}+ \\
+\frac{f}{\delta}\left\{-\left(\frac{2 \pi}{a}\right)^{2} A_{11} \cos \frac{2 \pi x}{a}-\left(\frac{2 \pi}{a}\right)^{2} A_{13} \cos \frac{2 \pi x}{a} \cos \frac{2 \pi y}{b}\right\}+\frac{N_{y}}{\delta} .
\end{gathered}
$$

As a result of solving the geometrically nonlinear problem by the Bubnov-Galerkin method using equality (7) and deflection (3), after cumbersome transformations, the following expression concerning the transverse compressive force can be obtained $N_{y}$

$$
\begin{gathered}
N_{y}=\left[\frac{4 D_{x} \pi^{2} b^{2}}{a^{4}}+\left(4 D+D_{x y}+D_{y x}\right) \frac{4 \pi^{2}}{3 a^{2}}+\frac{4 D_{y} \pi^{2}}{b^{2}}\right]+ \\
+\frac{4 \pi^{2} f}{3 a^{2}}\left[2\left(A_{11}+A_{12}-A_{13}\right)+2 f\left(A_{01}+A_{02}-A_{03}+0.5 A_{04}+0.5 A_{05}-A_{06}-A_{07}\right)\right]- \\
-\frac{16 \pi^{2} C_{x y} b^{2}}{3 a^{4}}\left[\left(A_{03}-2 A_{01}\right) f+\left(A_{13}-2 A_{11}\right)\right]-\frac{16 \pi^{2} C_{y x}}{3 b^{2}}\left[\left(A_{03}-2 A_{02}\right) f+\left(A_{13}-2 A_{12}\right)\right]- \\
-\frac{16 \pi^{2}}{3 a^{2}}\left(C_{x}+C_{y}-2 C\right)\left[A_{13}+f A_{03}\right]+\frac{\pi^{2} b^{3}}{4 R a^{3}}\left[\left(A_{03}-2 A_{01}\right) f+\left(A_{13}-2 A_{11}\right)\right]
\end{gathered}
$$


To determine the critical forces of the composite panel of asymmetric structure at $f \rightarrow 0$ we have

$$
\begin{aligned}
N_{y}= & {\left[\frac{4 D_{x} \pi^{2} b^{2}}{a^{4}}+\left(4 D+D_{x y}+D_{y x}\right) \frac{4 \pi^{2}}{3 a^{2}}+\frac{4 D_{y} \pi^{2}}{b^{2}}\right]-\frac{16 \pi^{2} C_{x y} b^{2}}{3 a^{4}}\left(A_{13}-2 A_{11}\right)-} \\
& -\frac{16 \pi^{2} C_{y x}}{3 b^{2}}\left(A_{13}-2 A_{12}\right)-\frac{16 \pi^{2}}{3 a^{2}}\left(C_{x}+C_{y}-2 C\right) A_{13}+\frac{\pi^{2} b}{4 R a}\left(A_{13}-2 A_{11}\right) .
\end{aligned}
$$

The obtained equality shows the influence of the radius $R$ on the critical force for the cylindrical panel, at which the considered delamination of an asymmetric structure loses its stability.

\section{Applied research}

Let us consider as an example the results (Table 1) of calculation of critical transverse forces $N_{y}$ calculated by formula (10) for delaminations with thickness $\delta=0.5 \mathrm{~mm}$, geometrical dimensions $a^{*} b=20 * 20 \mathrm{~mm}$ without considering the curvature and material characteristics corresponding to carbon fiber-reinforced plastic of KMU-4 type.

Table 1

\section{Critical forces of composite panels of asymmetric structure in compression}

\begin{tabular}{|c|c|c|c|}
\hline Laying & $\boldsymbol{N}_{\boldsymbol{y}}^{(\text {exac })}, \mathbf{k g f} \cdot \mathbf{m m}^{-2}$ & $\boldsymbol{N}_{\boldsymbol{y}}^{(\text {symm })} \mathbf{\text { kgf· } \mathbf { m m } ^ { - 2 }}$ & $\begin{array}{c}\text { Comparative } \\
\text { coefficients }\end{array}$ \\
\hline $45^{\circ} / 0^{\circ}$ & 70.09 & 71.53 & 0.98 \\
\hline $0^{\circ} / 90^{\circ}$ & 13.45 & 13.45 & 1.0 \\
\hline $45^{\circ} / 90^{\circ}$ & 11.85 & 13.6 & 0.87 \\
\hline $0^{\circ} / 45^{\circ} / 90^{\circ}$ & 11.52 & 11.72 & 0.98 \\
\hline $0^{\circ} / 90^{\circ} / 45^{\circ}$ & 20.85 & 17.38 & 1.2 \\
\hline $45^{\circ} / 0^{\circ} / 90^{\circ}$ & 24.75 & 27.11 & 0.91 \\
\hline
\end{tabular}

In Table 1 , the values of $N_{y}$ are determined by the obtained formula (10), and the values of $N_{y}^{(\text {symm })}$ consider only the terms related to the orthotropic structure without considering the mixed stiffnesses. With a detailed study of the results, in some cases the possible error of calculations for orthotropic panels exceeds $5 \%$.

\section{Conclusions}

1. The obtained solutions of the problems of stability and determining the carrying capacity of cylindrical composite panels of asymmetric structure under compression in the transverse direction can be used in determining the stress-strain state under geometrically nonlinear behaviour of thin delaminations.

The obtained results show the expediency of considering asymmetric structure for smooth panels even during static calculations, since the possible error of calculations according to the relations for orthotropic panels exceeds $5 \%$.

\section{Acknowledgements}

This work has been supported by the European Regional Development Fund within 1.1.1. Measure 1.1.1.2 "Aid to Post-Doctoral Research" of the Specific Aid Objective "To increase the research and innovative capacity of scientific institutions of Latvia and the ability to attract external financing, investing in human resources and infrastructure" of the Operational Programmed "Growth and Employment" (No. 1.1.1.2/VIAA/1/16/104 Structural Health Monitoring system for Inflight Monitoring (FLY SAFE).
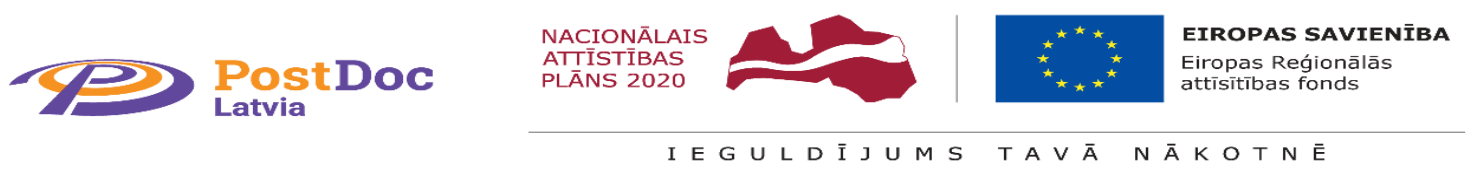


\section{References}

[1] Advisory Circular 20-107B, U.S. Department of Transportation Federal Aviation Administration, 2009.

[2] Urbahs A., Banovs M., Turko V., Urbaha M., Nedelko D., Lebedevs I. Research into the features of service damage in the composite material of helicopter rotor blades. Transport Means Proceedings of the International Conference, 2019-October, 2019, pp. 466-469.

[3] Chermoshentseva A.S., Pokrovskiy A.M., Bokhoeva L.A. The behavior of delaminations in composite materials - experimental results. IOP Conf. Series: Materials Science and Engineering, 2016, 116012005 DOI:10.1088/1757-899X/116/1/012005.

[4] Bokhoeva L A and Bochektueva E B 2019 An experimental research of the laminated composite plates stability. IOP Conf. Series: Materials Sci. and Eng. III Int. Conf. of Young Scientists on Contemporary Problems of Materials and Constructions pp. 012-027.

[5] Vasiliev V. V., Morozov E. V. Advanced Mechanics of Composite Materials and Structures. Amsterdam: Elsevier, 2018, 900 p.

[6] Mitrofanov O. V. Design of Carrying Panels of Aircraft Structures by Post-buckling Condition. MOSCOW: MAI, 2020, $160 \mathrm{p}$.

[7] Sørensen S. V., Zaitsev G. P. Bearing capacity of thin-walled structures made of reinforced plastics with defects. -Kiev: Naukova Dumka, 1982, 296 p.

[8] Abramovich H., Grunwald A., Pevsner P., Weller T., David A., Ghilai G., Green A., Peker N. Experiments on Axial Compression Postbuckling Behavior of Stiffened Cylindrical Composite Panels. 44th AIAA/ASME/ASCE/AHS/ASC Structures, Structural Dynamics, and Materials Conference. 5. 10.2514/6, 2003, pp. 2003-1793.

[9] Orifici A, Thomson R.S., Degenhardt R., Kling A., Rohwer K., Bayandor J. Degradation investigation in a postbuckling composite stiffened fuselage Panel. Composite Structures. 82, 2003, pp. 217-224. DOI: 10.1016/j.compstruct.2007.01.012.

[10] Ji R., Zhao L., Wang K., Liu F., Yu G., Zhang J. Effects of debonding defects on the postbuckling and failure behaviors of composite stiffened panel under uniaxial compression. Composite Structures. 256. 113121. 10.1016/j.compstruct.2020.113121.

[11] Mitrofanov O. V. Actual tasks and basic relations for the studies of composite panels of asymmetric structure in view of geometric nonlinearity // "Natural and Technical Sciences", -Moscow: Publishing House "Sputnik+", 2021. No.2. 